\title{
Scutellarin Attenuates Hypertension-Induced Expression of Brain Toll-Like Receptor 4/Nuclear Factor Kappa B
}

\author{
Xingyong Chen, ${ }^{1,2,3}$ Xiaogeng Shi, ${ }^{4}$ Xu Zhang, ${ }^{2}$ Huixin Lei, ${ }^{2}$ Simei Long, ${ }^{1,3}$ \\ Huanxing Su, ${ }^{5}$ Zhong Pei, ${ }^{1,3}$ and Ruxun Huang ${ }^{1,3}$ \\ ${ }^{1}$ Department of Neurology, National Key Clinical Department and Key Discipline of Neurology, \\ The First Affiliated Hospital Sun Yat-Sen University, Guangzhou 510080, China \\ ${ }^{2}$ Department of Neurology, Fujian Provincial Hospital, Fujian Medical University, Fuzhou 350001, China \\ ${ }^{3}$ Guangdong Key Laboratory for Diagnosis and Treatment of Major Neurological Diseases, The First Affiliated Hospital, \\ Sun Yat-Sen University, Guangzhou 510080, China \\ ${ }^{4}$ Department of Neurology, The Second Affiliated Hospital, Guangzhou University of Chinese Medicine, \\ Guangzhou 510120, China \\ ${ }^{5}$ Key Laboratory of Quality Research in Chinese Medicine, Institute of Chinese Medical Sciences, University of Macau, \\ Taipa 999078, Macau
}

Correspondence should be addressed to Xiaogeng Shi; sxghome@aliyun.com and Zhong Pei; peizhong@mail.sysu.edu.cn

Received 2 May 2013; Accepted 13 August 2013

Academic Editor: Geeta Ramesh

Copyright ( $\odot 2013$ Xingyong Chen et al. This is an open access article distributed under the Creative Commons Attribution License, which permits unrestricted use, distribution, and reproduction in any medium, provided the original work is properly cited.

Hypertension is associated with low-grade inflammation, and Toll-like receptor 4 (TLR4) has been shown to be linked to the development and maintenance of hypertension. This study aimed to investigate the effects of scutellarin (administered by oral gavage daily for 2 weeks) on brain TLR4/nuclear factor kappa B-(NF- $\kappa$ B-) mediated inflammation and blood pressure in renovascular hypertensive (using the 2-kidney, 2-clip method) rats. Immunofluorescence and western immunoblot analyses revealed that hypertension contributed to the activation of TLR4 and NF- $\kappa \mathrm{B}$, accompanied by significantly enhanced expression of proinflammatory mediators, such as tumor necrosis factor- $\alpha$ (TNF- $\alpha$ ), interleukin-1 $\beta$ (IL-1 $\beta$ ), and interleukin-18 (IL-18). Furthermore, expression of the antiapoptotic protein, myeloid cell leukemia-1 (Mcl1), was decreased, and the pro-apoptotic proteins, Bax and cleavedcaspase3 p17 were increased in combined cerebral cortical/striatal soluble lysates. Scutellarin significantly lowered blood pressure and attenuated the number of activated microglia and macrophages in brains of hypertensive rats. Furthermore, scutellarin significantly reduced the expression of TLR4, NF- $\kappa$ B p 65 , TNF- $\alpha$, IL- $1 \beta$, IL-18, Bax and cleaved-caspase- 3 p17, and increased the expression of Mcll. Overall, these results revealed that scutellarin exhibits anti-inflammatory and anti-apoptotic properties and decreases blood pressure in hypertensive rats. Therefore, scutellarin may be a potential therapeutic agent in hypertension-associated diseases.

\section{Introduction}

Hypertension is a major risk factor for cardiovascular accidents [1] and may also accelerate the onset and progression of ischemia stroke and cerebral hemorrhage [2]. Hypertension is associated with low-grade inflammation, which may be associated with hypertension-mediated damage to target organs [3]. Inflammation in brain parenchyma can occur as a local process that can be triggered and sustained by activated glial cells, which is thought to contribute to the pathogenesis of several diseases [3]. The innate immune response, predominantly represented by Toll-like receptors (TLRs), has been shown to contribute to the development of this condition [4].

TLRs are first-line molecules for initiating the innate immune responses, and thus its signaling is involved in the activation of microglia by pathogens and damaged host cells. Activation of microglia by TLRs is considered to be the "classical" form of activation [5]. Activated microglia subsequently secret proinflammatory cytokines and express costimulatory molecules needed for protective immune responses to pathogens and efficient clearance of damaged tissues [6]. 
TLR4 is an important contributor to microglial activation and known to initiate an inflammatory cascade in response to various brain injuries [7]. TLR4 has been shown to be linked to the development and maintenance of hypertension [8]. Furthermore, TLR4 is involved in cerebrovascular diseases, including stroke [9] and neurodegeneration [10]. TLR4mediated signaling activates the nuclear factor kappa B (NF$\kappa \mathrm{B})$ signaling pathway, which plays a critical role in immune and inflammatory responses, cell death, and survival $[5,9]$. Therefore, enhanced expression of brain TLR4/NF- $\kappa \mathrm{B}$ may play an important role in cerebral pathology induced by hypertension.

5,6,4-Trihydroxyflavone-7-O-glucoronide (scutellarin) is a flavone and the major active component of Erigeron breviscapus (Vant.) Hand-Mazz, a herbal medicine in use in the Orient for the treatment of cerebrovascular diseases [11-13]. In recent years, many studies in different animals and cells models have provided evidence for the protective effects of scutellarin because of its antioxidant $[12,14,15]$, antiapoptotic $[14,16-18]$, anti-inflammatory $[19,20]$, and calcium channel antagonist properties [21]. Therefore, the aim of the present study was to investigate whether scutellarin treatment reduced the expression of brain TLR $4 / N F-\kappa B$, the inflammatory status, and blood pressure in renovascular hypertensive rats.

\section{Materials and Methods}

2.1. Animals. All experimental procedures were approved by the Institutional Animal Ethical Committee of Sun Yat-Sen University and were conducted according to the Guide for the Care and Use of Laboratory Animal of the National Institute of Health (Publication no. 80-23, revised 1996). A total of 24 male Sprague-Dawley rats (60-80 g) were purchased from the Center for Experimental Animals of Sun Yat-Sen University. Rats were randomly assigned into four groups (six rats per group): (1) sham-operated group (normotensive controls), (2) hypertension with normal saline (NS) treatment, (3) hypertension with low-dose (5 mg/kg per day) scutellarin, and (4) hypertension with high-dose $(20 \mathrm{mg} / \mathrm{kg}$ per day) scutellarin. Scutellarin Scutellarin (Yunnan Biovalley pharmaceutical Company Ltd, Yunnan, China) was dissolved in sterile NS, and different doses of scutellarin were administered by gavage lasting for 2 weeks.

2.2. Hypertension Model and Drug Administration. Hypertension was induced using a 2-kidney, 2-clip method (2K2C), as described by Zeng et al. [22]. Briefly, under anesthesia with $10 \%$ chloral hydrate $(3 \mathrm{~mL} / \mathrm{kg}$ body weight, intraperitoneally [i.p.]), a median longitudinal incision on the abdominal skin was performed, and then the roots of both right and left renal arteries were constricted by placing ring-shaped silver clips with an inner diameter of $0.30 \mathrm{~mm}$ to induce hypertension. Approximately 8 weeks later, those rats with systolic blood pressure higher than $140 \mathrm{mmHg}$ and without stroke symptoms were selected for the experiment. Different doses of scutellarin were administered by gavage daily for 2 weeks. In the NS group, hypertensive rats were given saline in the same volume as scutellarin. Renal arteries in shamoperated rats also underwent surgery but without placement of clips. Systolic blood pressure (SBP) was measured by an indirect tail-cuff sphygmomanometer (MRB-IIIA, Shanghai Institute of Hypertension, Shanghai, China) in conscious rats heated (heat lamp at $37^{\circ} \mathrm{C}$, for $5 \mathrm{~min}$ ) before and after renal artery constriction (at weekly intervals) for 10 weeks [23].

2.3. Preparation of Tissue Samples. The preparation of tissue samples was performed as described previously [24, 25]. Briefly, after 2 weeks of scutellarin treatment, rats were sacrificed under deep anesthesia with $10 \%$ chloral hydrate ( $5 \mathrm{~mL} / \mathrm{kg}$ body weight, i.p.) and then transcardially perfused with $0.9 \%$ sodium chloride (at $4^{\circ} \mathrm{C}$ ). The brains were removed, and the left frontal cerebral cortex and striatum were rapidly dissected and used for western immunoblotting analysis. For immunofluorescence labeling, the right frontal brain was sliced into horizontal sections (10 $\mu \mathrm{m}$ thick) using the CM1900 cryostat (Leica, Heidelberg, Germany), and these section, were then fixed with $4 \%$ paraformaldehyde (in 0.01 M phosphate-buffered saline (PBS), pH 7.4).

2.4. Immunofluorescence Labeling. Immunofluorescence was carried out as described previously [7]. Briefly, sections were preincubated with $0.3 \%$ Triton X-100 in $0.01 \mathrm{M}$ PBS (10 min), blocked with $10 \%$ normal goat serum (KPL, CA, USA) (1 h at room temperature RT), and then incubated (overnight at $4^{\circ} \mathrm{C}$ ) with the primary antibodies (in primary antibody diluents (Dako, Denmark)): rabbit anti-TLR4 (1:100) (Santa Cruz Biotechnology, Santa Cruz, CA, USA), mouse anti-Neuronal Nuclei (NeuN) (1:400) (Chemicon, USA), mouse anti-rat glial fibrillary acid protein (GFAP) (1:800) (Cell Signaling Technology, Beverly, MA, USA), or mouse anticluster of differentiation 11b (CD11b or OX-42) (1:300) (Millipore, USA). Sections were then incubated with Alexa Fluor 555 conjugated goat anti-rabbit $\operatorname{IgG}(\mathrm{H}+\mathrm{L}), \mathrm{F}\left(\mathrm{ab}^{\prime}\right) 2$ Fragment (1:1000), Alexa Fluor 555 conjugated goat anti-mouse IgG $(\mathrm{H}+\mathrm{L}), \mathrm{F}\left(\mathrm{ab}^{\prime}\right) 2$ Fragment $(1: 1000)$ (both from Cell Signaling Technology), or fluorescein isothiocyanate-goat anti-mouse IgG antibodies $(1: 200)$ (Zymed, USA) in $0.01 \mathrm{M}$ PBS ( $1 \mathrm{~h}$ at RT). Sections counterstained for nuclei were exposed to $4^{\prime}, 6$ diamidino-2-phenylindole dihydrochloride (1:1000) (Roche, Mannheim, Germany) and then mounted in ProLong Gold antifade reagent (P36930, Invitrogen, USA) prior to imaging. Immunoreactivity was visualized using the BX51 microscope (Olympus). Negative control sections were incubated with PBS only, and showed no positive staining (data not shown).

2.5. Western Immunoblot Analyses. Western immunoblotting was performed as previously described [26]. Briefly, the left frontal cerebral cortex and striatum were homogenized in lysis buffer ( $\mathrm{pH} 7.6$ ) with $0.01 \mu \mathrm{g} / \mathrm{mL}$ phenylmethanesulfonyl fluoride centrifuged (16,400 rpm for $30 \mathrm{~min})$, and then Protein concentrations were determined using a bicinchoninic acid (BCA) protein assay kit (PIERCE, USA) according to the manufacturer's instructions. Soluble protein $(50 \mu \mathrm{g})$ was separated by $4-20 \%$ gradient SDS/PAGE (sodium dodecyl sulfate polyacrylamide gel electrophoresis) (Bio-Rad) and then transferred onto polyvinylidene fluoride membranes 
(Millipore, USA). Membranes were blocked with 5\% skim milk in Tris-buffered saline containing 0.1\% Tween-20 and then incubated with the primary antibodies: rabbit antiTLR4 (1:1000), rat anti-interleukin 1 beta (IL-1 $\beta)(1: 1000)$, rabbit anti-myeloid cell leukemia-1 (mcl1) (1:1000) (all from Abcam, USA), mouse anti-NF- $\kappa$ B p65 (1:1000) (Cell Signaling Technology, USA), goat anti-tumor necrosis factor alpha (TNF- $\alpha)(1: 5000)$ (Novus Biologicals, USA), rabbit anti-IL$18(1: 300)$, rabbit anti-Bax $(1: 300)$, or mouse anti-caspase3 pl7 (1:300) (all from Santa Cruz Biotechnology, USA). Membranes were exposed to the secondary antibodies diluted in blocking buffer for $1 \mathrm{~h}$ at room temperature: horseradish peroxidase (HRP)-conjugated goat anti-mouse $(1: 6000)$ (EarthOx, USA), HRP-conjugated goat anti-rabbit $(1: 3000)$ (Cell Signaling Technology), or HRP-conjugated rabbit antigoat IgG antibodies $(1: 3000)$ (Invitrogen, USA). Mouse monoclonal anti- $\beta$-actin $(1: 3000)$ (Proteintech Group Inc., USA) served as the housekeeping protein. Immunoreactive bands were detected using Chemiluminescent HRP Substrate (Millipore, USA) and visualized on Kodak X-OMAT films. The optical densities were normalized to those of $\beta$-actin and calculated as target protein expression $/ \beta$-actin expression ratios (using Image J 1.42q).

2.6. Image Analysis and Quantification. All histological images were analyzed with Image-Pro Plus image analysis software (Media Cybernetics, Silver Spring, MD, USA) by one blinded assessor. The number of positively stained cells was counted using Image-Pro Plus image analysis software in nine comparable, nonoverlapping fields $(425 \mu \mathrm{m} \times 320 \mu \mathrm{m} ; 3$ fields per section $\times 3$ sections per rat) and was presented as the average cell number per field on each section $[27,28]$.

2.7. Statistical Analysis. All data are expressed as the mean \pm standard deviation and were analyzed by one-way analysis of variance (ANVOA) followed by the least significant difference (LSD) post hoc test. Significance was reached at values of $P<0.05$ and $P<0.001$. Statistical analysis was performed with Statistical Product and Service Solutions (SPSS) 13.0 (SPSS Inc., Chicago, IL, USA).

\section{Results}

3.1. Effect of Scutellarin Treatment on SBP. Baseline SBP was similar between the four groups. SBP was only slightly increased in hypertension-induced rats but increased progressively to $174.7 \pm 13.9,180.9 \pm 6.2,178.8 \pm 6.7$, and $126.4 \pm$ $9.8 \mathrm{mmHg}$ in NS, low-dose, high-dose, and sham-operated groups, respectively (Figure 1). SBP in the NS, low-dose, and high-dose groups was significantly higher compared with the sham-operated group $(P<0.001)$. There were no incidences of stroke or death in the four groups (Table 1). No significant difference in SBP was found before treatment in NS, low-dose and high-dose groups. Compared with the NS group (196.5 \pm $9.8 \mathrm{mmHg}$ ), scutellarin treatment significantly reduced SBP in a dose-dependent manner $(P<0.001)$. In the low-dose group, SBP was decreased by approximately $11.5 \pm 6.5 \mathrm{mmHg}$, from $180.9 \pm 6.2 \mathrm{mmHg}$ to $169.1 \pm 7.1 \mathrm{mmHg}$. In the high-dose group, SBP was reduced by approximately $17.2 \pm 7.4 \mathrm{mmHg}$,

\begin{tabular}{cccc}
\hline Group & Baseline & Before treatment & After treatment \\
\hline Sham & $102.7 \pm 7.8$ & $126.4 \pm 9.8$ & $137.2 \pm 8.3$ \\
NS & $99.4 \pm 7.0$ & $174.7 \pm 13.9$ & $196.5 \pm 9.8$ \\
Low dose & $100.2 \pm 6.8$ & $180.9 \pm 6.2$ & $169.1 \pm 7.1$ \\
High dose & $101.4 \pm 5.9$ & $178.8 \pm 6.7$ & $161.2 \pm 9.9$ \\
\hline
\end{tabular}

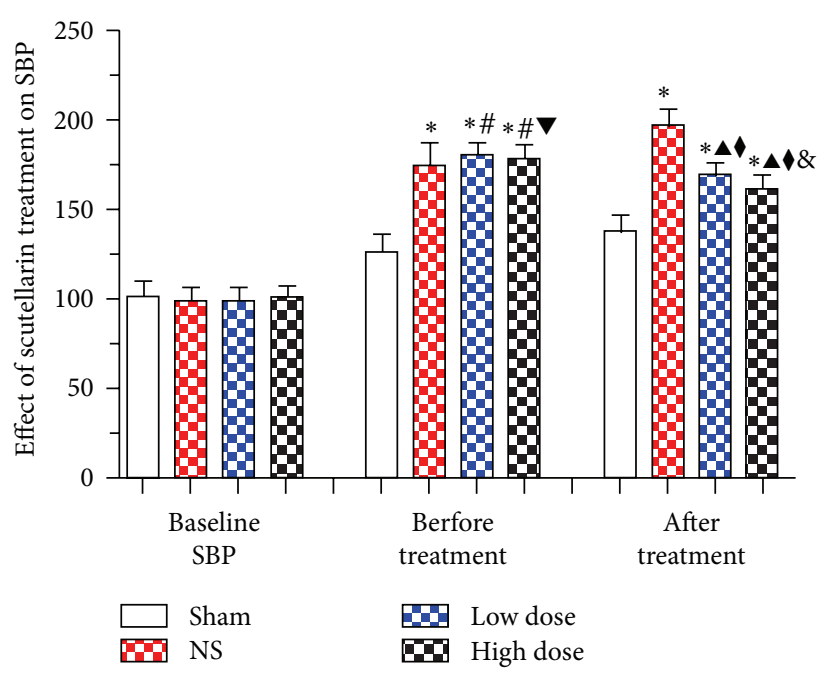

FIGURE 1: Effect of scutellarin treatment on systolic blood pressure (SBP). Baseline systolic blood pressure (SBP) ( $\mathrm{mmHg}$ ) was similar among the four experimental groups. SBP was significantly increased in hypertensive groups compared with the sham-operated group. No significant difference in SBP was found in NS rats prior to treatment. However, SBP was significantly reduced with low- and high-dose scutellarin. ${ }^{*} P<0.001$ versus sham group; ${ }^{\#} P>0.05$, ${ }^{\star} P<0.001$ versus NS group; ${ }^{\uparrow} P<0.001$, versus before treatment; ${ }^{\nabla} P>0.05,{ }^{\&} P<0.001$ versus low-dose group.

TABLE 1: Occurrence of stroke and death in different groups of rats. There were no incidences of stroke or death in the four groups.

\begin{tabular}{lccccc}
\hline Group & \multicolumn{5}{c}{ Stroke } \\
& $\mathrm{CH}$ & $\mathrm{CI}$ & $\mathrm{CH}+\mathrm{CI}$ & $\mathrm{SAH}$ & Death \\
\hline Sham & 0 & 0 & 0 & 0 & 0 \\
NS & 0 & 0 & 0 & 0 & 0 \\
Low dose & 0 & 0 & 0 & 0 & 0 \\
High dose & 0 & 0 & 0 & 0 & 0 \\
\hline
\end{tabular}

$\mathrm{CH}$ : cerebral hemorrhage; $\mathrm{CI}$ : cerebral infarction; SAH: subarachnoid hemorrhage.

from $178.8 \pm 6.7 \mathrm{mmHg}$ to $161.2 \pm 9.9 \mathrm{mmHg}$. Furthermore, SBP was significantly different between the low-dose and high-doses group $(P<0.001)$ and was significantly higher compared with the sham-operated group $(137.2 \pm 8.3 \mathrm{mmHg})$ $(P<0.001)$.

3.2. Scutellarin Decreased the Number of Activated Microglia/ Macrophages in Hypertensive Rats. OX-42 labels both microglia and macrophages. Chronic hypertension induced 


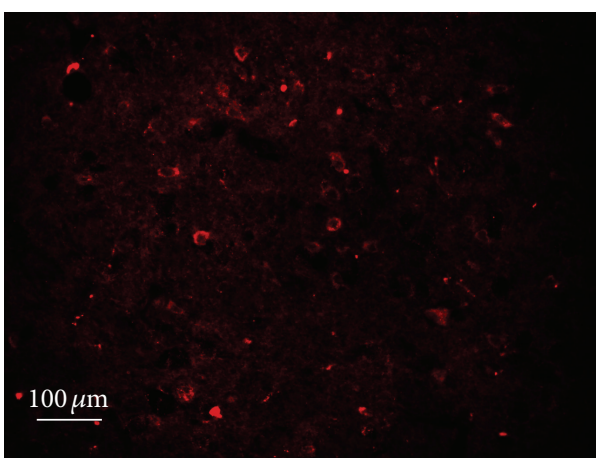

(a)

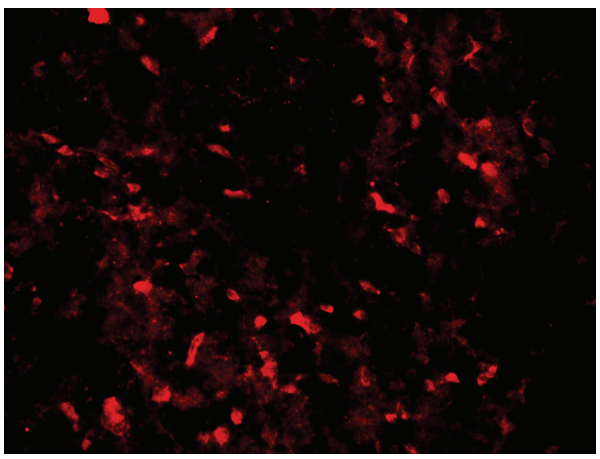

(c)

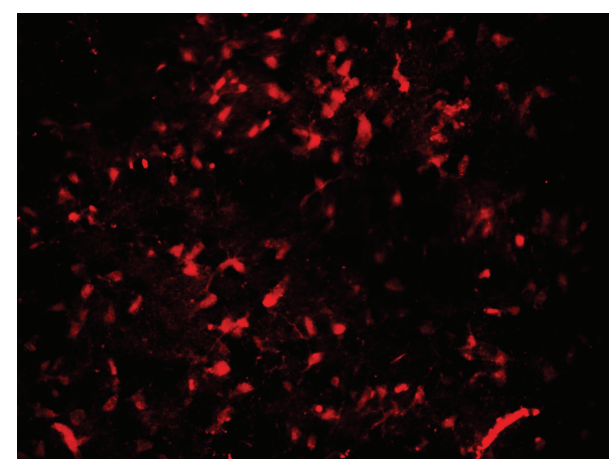

(b)

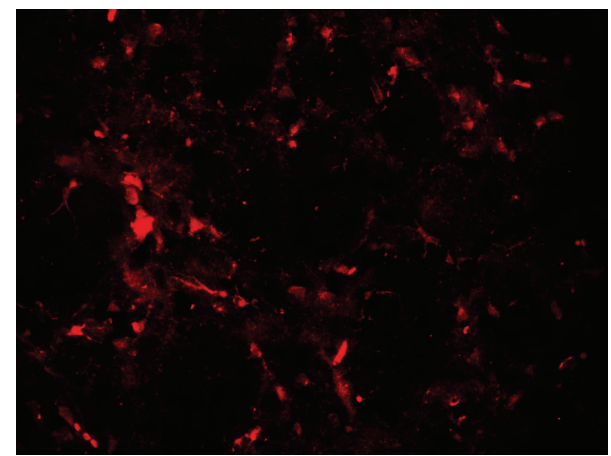

(d)

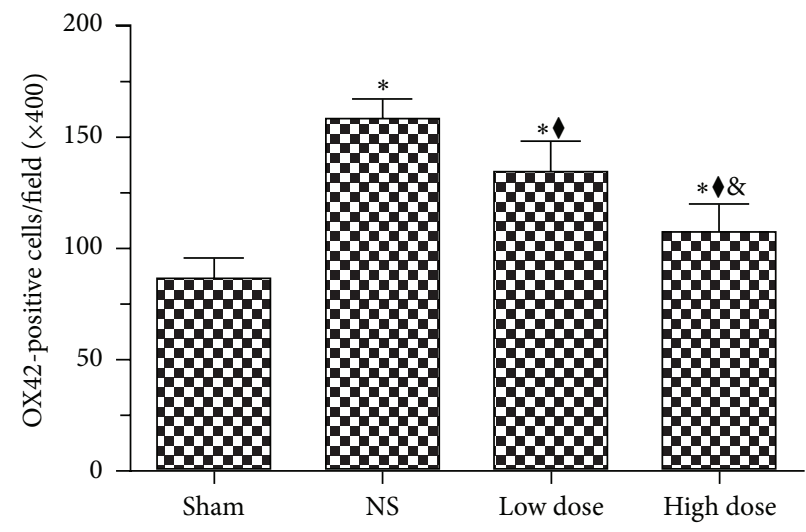

(e)

FIGURE 2: Scutellarin decreases the number of activated microglia in brain of hypertension. (a-d) Cluster of differentiation 11b (OX-42) immunostaining for microglia. (e) Quantification of OX-42-positive cells revealed that activated microglia were significantly increased in normal saline (NS) rats compared with the sham group, and treatment with scutellarin significantly reduced these numbers in a dosedependent manner. ${ }^{*} P<0.001$ versus sham group; ${ }^{\circledR} P<0.001$ versus NS group; ${ }^{\&} P<0.001$ versus low-dose group. Scale bar $=100 \mu \mathrm{m}$.

microglia/macrophage activation (Figure 2). Compared with the sham group, the number of cells positively stained with OX-42 was significantly increased in the NS group $\left(178.7 \pm 18.5 / \mathrm{mm}^{2}\right.$ versus $\left.86.2 \pm 16.8 / \mathrm{mm}^{2}\right)(P<0.001)$. In contrast, the number of cells positively stained with OX-42 was significantly decreased with low-dose and high-dose scutellarin, $143.1 \pm 21.9 / \mathrm{mm}^{2}$ and $117.4 \pm 17.8 / \mathrm{mm}^{2}$, respectively $(P<0.001)$. Furthermore, counts in the high-dose group were significantly lower compared with the low group $(P<0.001)$.

3.3. Scutellarin Suppressed Hypertension-Induced Expression of Brain TLR4. TLR4 immunoreactivity was sparse in the cerebral cortex and striatum in sham-operated rats (Figure 3(a); sham). In contrast, chronic hypertension induced higher TLR4 expression in these regions (Figure 3(a); hypertension). TLR4 was further investigated for its cellular distribution using markers for neurons $(\mathrm{NeuN})$, astrocytes (GFAP) and microglia (OX-42). The majority of TLR4 (red) was colabeled with OX-42-positive cells (green) (Figure 3(b); (A)). In contrast, few TLR4positive cells were co-labeled with GFAP-positive (green) (Figure 3(b); (B)) or NeuN-positive (green) (Figure 3(b); (C)) cells. Western immunoblot analysis showed that compared with the sham-operated group, levels of TLR4 protein were significantly increased (approximately 6-fold) in the 


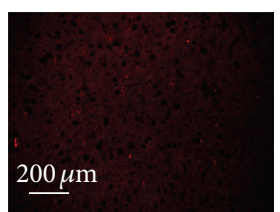

Sham

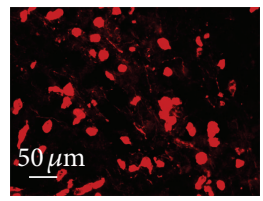

TLR4

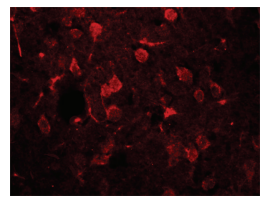

TLR4

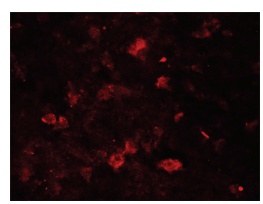

TLR4

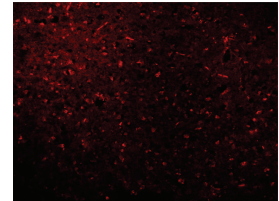

Hypertension

(a)

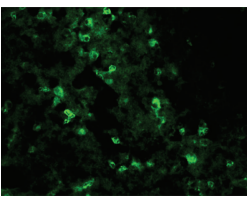

OX42

(A)

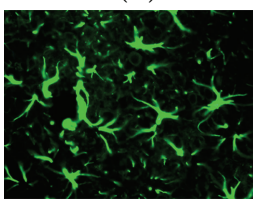

GFAP

(B)

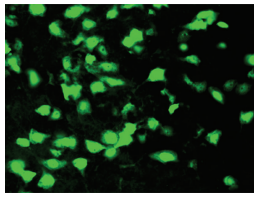

NEUN
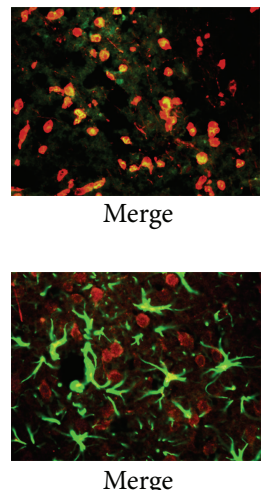

Merge

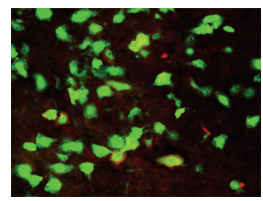

Merge

(C)

(b)
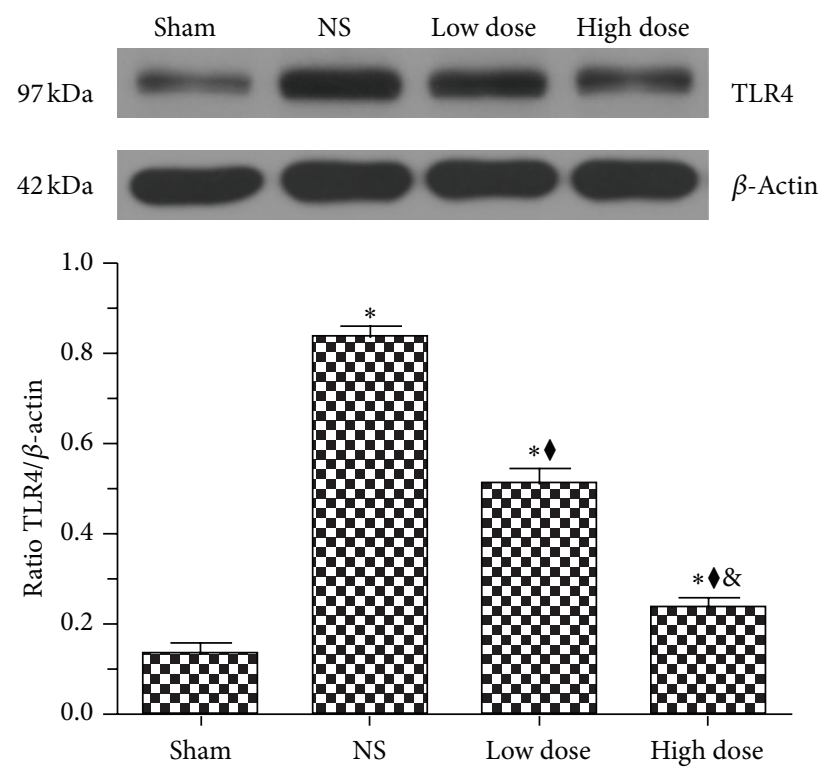

(c)

FIGURE 3: Scutellarin suppresses hypertension-induced expression of brain Toll-like receptor 4 (TLR4). (a) Chronic hypertension significantly enhanced the expression of TLR4 in the cerebral cortex. (b) Double immunofluorescence labeling indicated that the majority of TLR4 (red) was colabeled with OX-42-positive (green) cells and fewer with glial fibrillary acid protein- or Neuronal Nuclei-positive cells. (c) Western immunoblot analysis of brains of hypertensive rats showed that scutellarin dose-dependently suppressed the expression of TLR4. ${ }^{*} P<0.001$ versus sham group; ${ }^{\dagger} P<0.001$ versus NS group; ${ }^{\circledR} P<0.001$ versus low-dose group. (c) Scale bar $=200 \mu \mathrm{m}$ (a) and $50 \mu \mathrm{m}$ (b).

NS group (Figure 3(c)) $(P<0.001)$. However, treatment with scutellarin significantly decreased this level in a dosedependent manner, approximately $39.9 \%$ and $72.1 \%$ in the low-dose and high-dose groups, respectively (Figure 3(c)) $(P<0.001)$.

\subsection{Scutellarin Attenuated Hypertension-Induced Expression} of $N F-\kappa B, T N F-\alpha, I L-1 \beta$, and IL-18. TLR4 mediates the activation of transcription factors, such as NF- $\kappa \mathrm{B}$, which subsequently induces the production of inflammatory cytokines. In the present study, western immunoblot analysis showed that compared with the sham group, protein levels of NF$\kappa \mathrm{B}$ p 65 , TNF- $\alpha$, IL-1 $\beta$, and IL-18 in NS rats were significantly increased by approximately 7-, 6.5-, 3.7-, and 4-fold, respectively (Figures $4(\mathrm{a})-4(\mathrm{~d}))(P<0.001)$. These proteins were significantly reduced by scutellarin, with the high-dose group inducing a markedly higher attenuation compared with the low-dose group (Figures 4(a)-4(d)) $(P<0.001)$. Levels of NF- $\kappa$ B p 65 , TNF- $\alpha$, IL- $1 \beta$, and IL-18 were reduced to $51.1 \%$, $61.2 \%, 82.9 \%$, and $83.3 \%$, respectively, in the low-dose group, in contrast to $31.4 \%, 41.9 \%, 57.8 \%$, and $53.4 \%$, respectively, in the high-dose group. Therefore, these results suggest that scutellarin reduced hypertension-mediated induction of the inflammatory response.

3.5. Scutellarin Treatment Upregulated the Expression of Mcll, and Suppressed Bax and Caspase-3 p17. To investigate the potential effect of scutellarin on neuronal cell survival, we evaluated the expression of the apoptosis-related proteins, Mcl1, Bax, and cleavedcaspase-3 p17 in brains of hypertensive and sham-operated rats. Western immunoblot analysis indicated that compared with the sham group, levels of Mcll, Bax, and cleavedcaspase- 3 p17 were significantly increased in hypertensive rats (Figures 5(a)-5(c)) $(P<0.001)$. Compared with sham rats, Mcll, Bax, and cleavedcaspase-3 p17 were significantly $(P<0.001)$ elevated in the NS group by approximately 2-, 3.8-, and 8.9-fold, respectively. Compared with the NS group, treatment with scutellarin significantly upregulated the level of Mcll, particularly with the high-dose group (approximately 2.1-fold compared with the NS group) (Figure 5(a)) $(P<0.001)$. However, scutellarin significantly downregulated the levels of Bax and cleavedcaspase-3 p17 protein in a dose-dependent manner, to $71.4 \%$ and $73.9 \%$ (for Bax and cleavedcaspase- 3 p17, resp.) for the low-dose group 


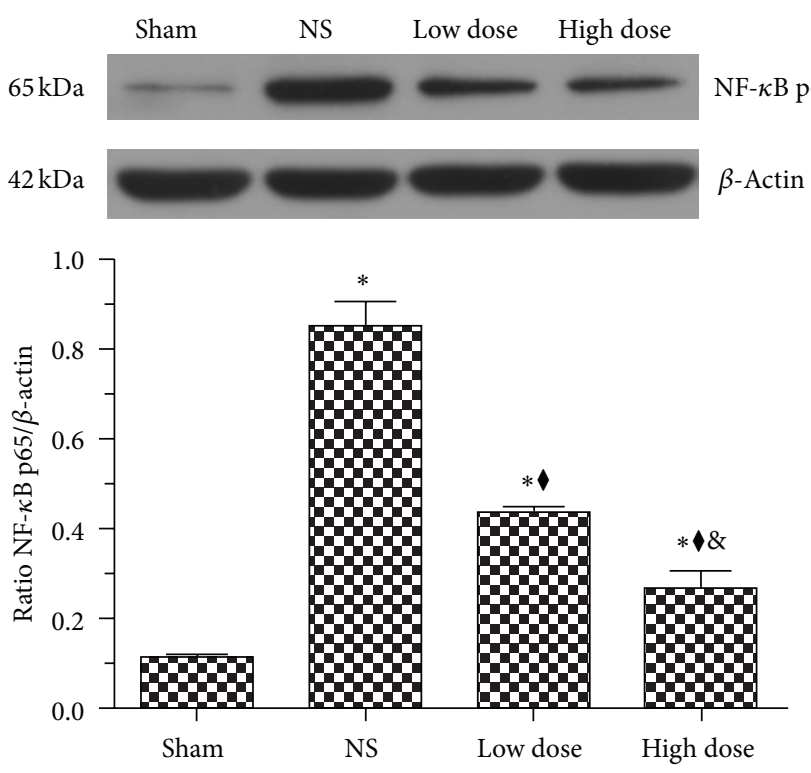

(a)
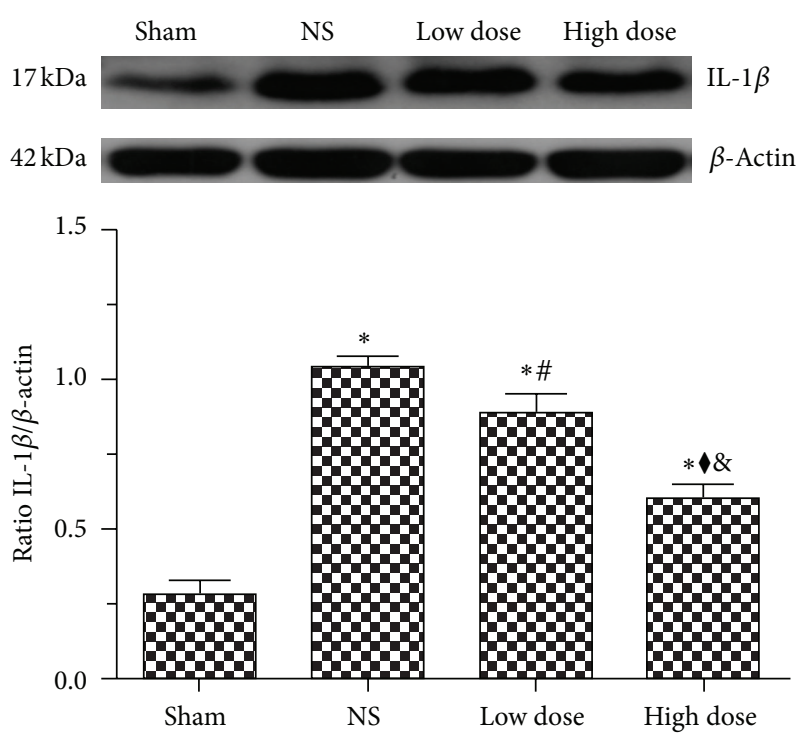

(c)
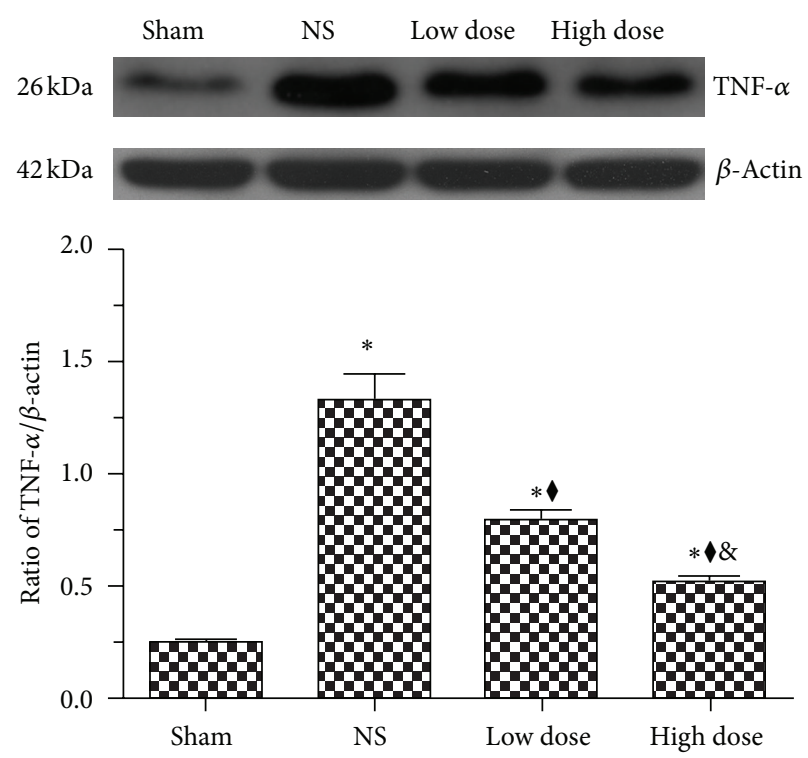

(b)
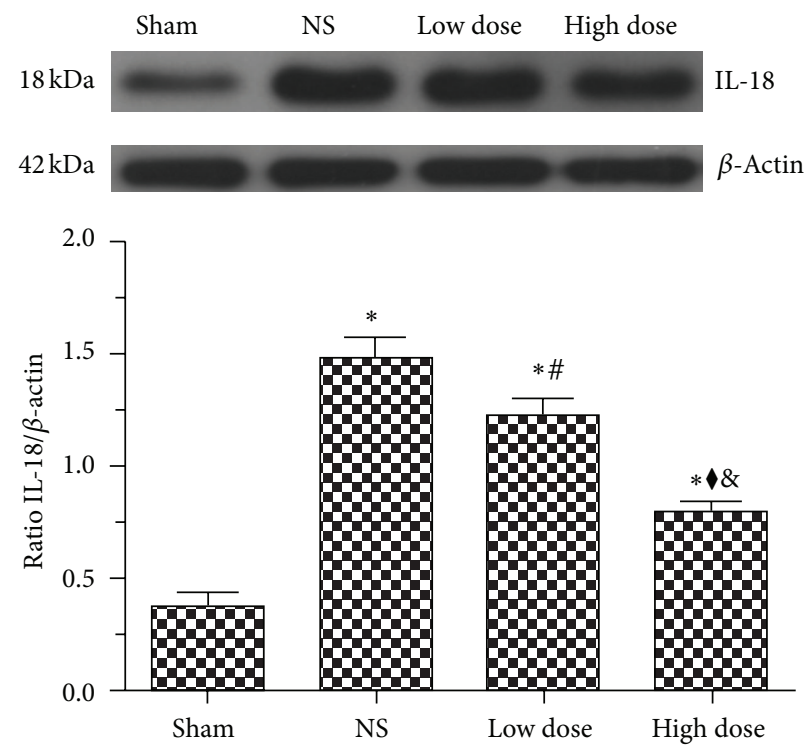

(d)

FIGURE 4: Scutellarin attenuates hypertension-induced brain expression of NF- $\kappa$ B, TNF- $\alpha$, IL-1 $\beta$, and IL-18. Western immunoblot analysis for (a) NF- $\kappa$ B p65, (b) TNF- $\alpha$, (c) IL-1 $\beta$, and (d) IL-18 in the rat cortex and striatum. Treatment with scutellarin significantly reduced the expression of these inflammatory markers in a dose-dependent manner. ${ }^{*} P<0.001$ versus sham group; ${ }^{\#} P<0.05,{ }^{*} P<0.001$ versus NS group; ${ }^{\&} P<0.001$ versus low-dose group.

and $56.3 \%$ and $27.1 \%$ (for Bax and cleavedcaspase-3 p17, resp.) for the high-dose group (Figures 5(a) and 5(c)) $(P<0.001)$.

\section{Discussion}

In the present study, we demonstrate that scutellarin is protective against chronic hypertension-induced activation of brain TLR4 and subsequent NF- $\kappa \mathrm{B}$-mediated inflammatory responses. We show that scutellarin possesses anti-inflammatory and antiapoptotic properties and lowers blood pressure, thus suggesting its use as a potential therapeutic agent in hypertension-associated diseases.
In the central nervous system, TLR4 has been reported to be expressed in both microglia and astrocytes, as well as in neurons [7]. In this study, chronic hypertension augmented the expression of TLR4 predominantly in microglia/ macrophage cells, indicating its involvement in chronic hypertension-induced inflammation. However, the expression of TLR4 in astrocytes and neurons also suggested their potential involvement, and thus further studies could explore this possible relationship.

Innate and adaptive immunities have been shown to contribute to hypertension-associated end-organ damage, although the mechanism by which this occurs remains 


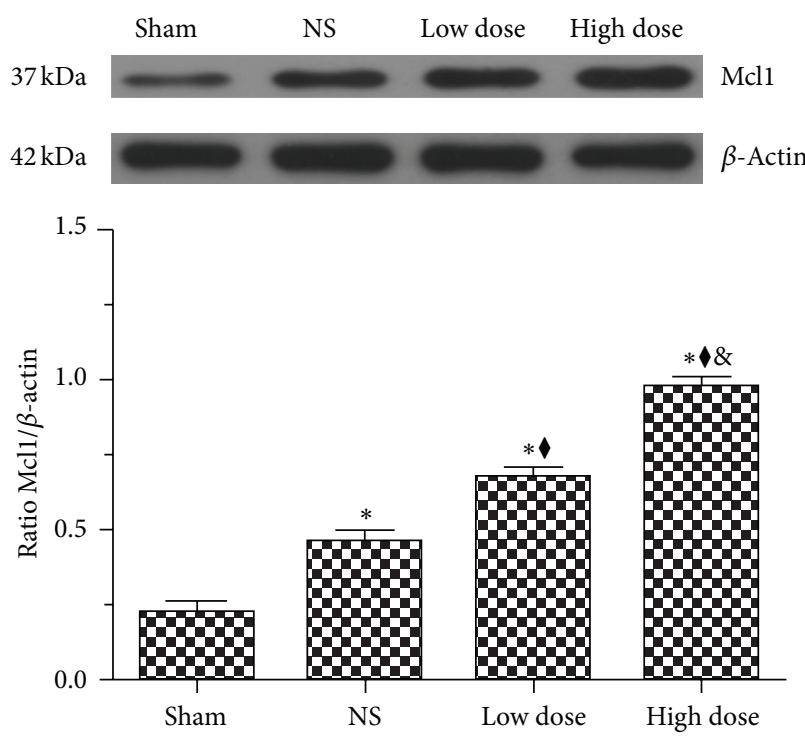

(a)
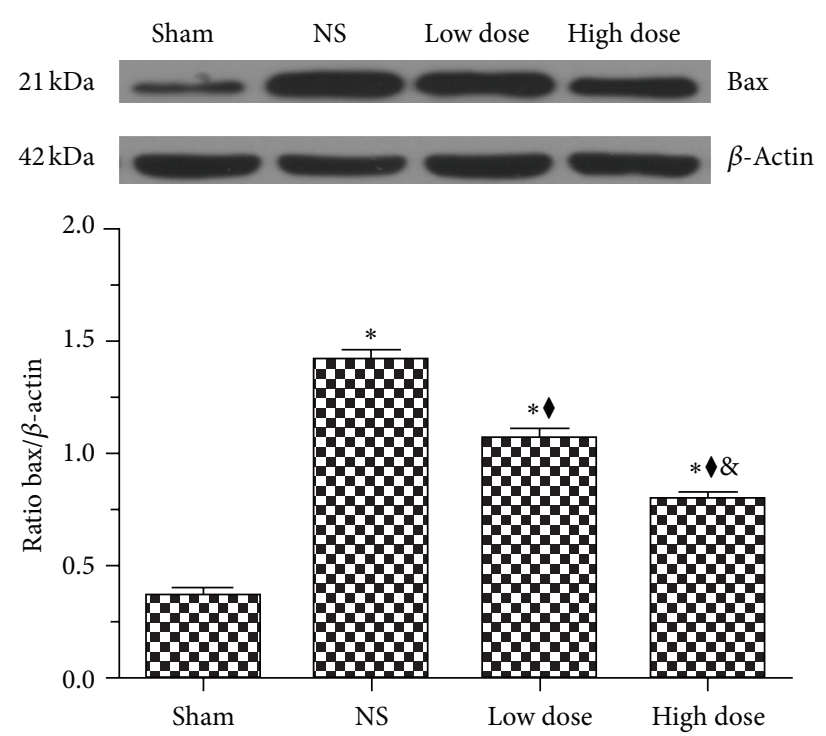

(b)
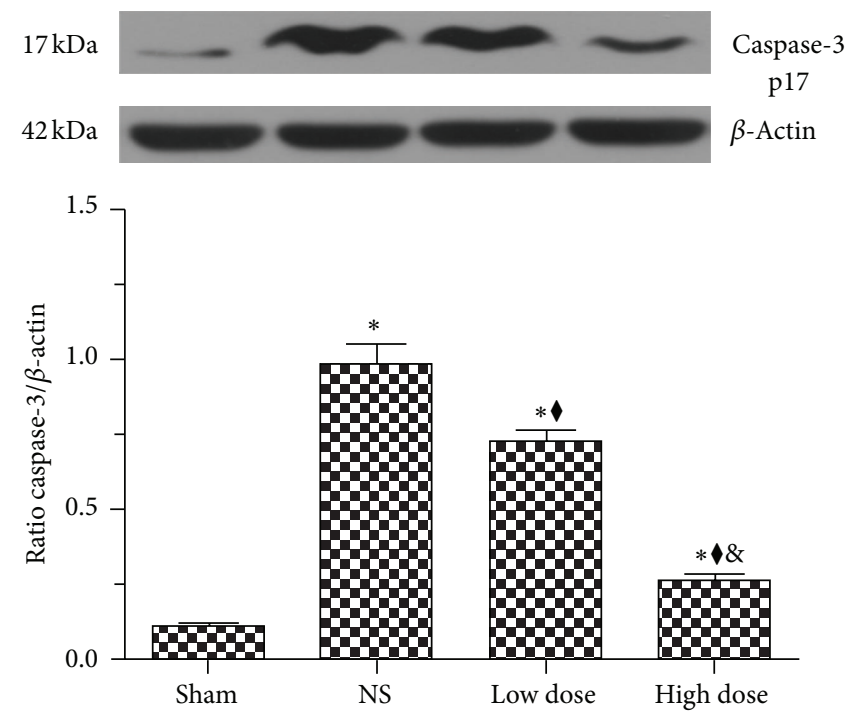

(c)

FIGURE 5: Scutellarin treatment upregulated Mcll and suppressed Bax, caspase-3 p17 expression. Western immunoblot analysis of protein levels of (a) Mcl1, (b) Bax, and (c) cleavedcaspase-3 p17 in the rat cortex and striatum. Scutellarin significantly upregulated Mcl1 but dosedependently decreased Bax and cleavedcaspase- 3 pl7 (c). ${ }^{*} P<0.001$ versus sham group; ${ }^{*} P<0.001$ versus NS group; ${ }^{\&} P<0.001$ versus low-dose group.

unclear [29]. Previous studies suggest that enhanced expression of TLR4 may be linked with the development and maintenance of hypertension and low-grade inflammation and augmented vascular contractility in hypertensive rats $[8,29]$. Chronic hypertension causes cardiac hypertrophy, characterized by low-grade inflammation and accompanied by increased expression and activity of TLR4, and elevated gene expression of TNF- $\alpha$ and IL- 6 in cardiac tissue [8]. Treatment with anti-TLR4 was shown to decrease mean arterial pressure, TLR4 protein in mesenteric resistance arteries, and serum levels of IL- 6 in spontaneously hypertensive rats [29]. Furthermore, TLR4 signaling is also involved in brain damage and in neuroinflammatory processes associated with ischemic stroke and neurodegenerative diseases, such as Alzheimer's disease [10, 30-32]. Neutralizing TLR4 at the time of intracerebral hemorrhage [7] and ischemic stroke [31] provides neuroprotection. This effect may result from TLR4mediated activation of NF- $\kappa$ B signaling pathways linked to the transcription of many proinflammatory genes encoding for cytokines, chemokines, proteins of the complement system, and cell adhesion molecules. Findings from our study of chronically hypertensive rats revealed that in addition to reducing blood pressure, scutellarin prevented inflammatory mediated neuronal damage by suppressing microglial activation and the concomitant rise in expression of NF- $\kappa \mathrm{B}$, TNF$\alpha$, IL-1 $\beta$, and IL-18. The underlying mechanism involves, at 
least in part, inhibition of TLR4/NF- $\kappa \mathrm{B}$-dependent signaling pathway. Interestingly, although treatment with scutellarin decreased SBP, the antihypertensive effect was moderate and without a dose-response relationship, suggesting that the low dose of scutellarin may have already reached the maximum antihypertensive effect. Thus, the antihypertensive action may play a minor role in the protective activity of scutellarin against hypertension-induced brain inflammation. Therefore, these results suggest that TLR4 is a promising target for the prevention and treatment of hypertension-associated diseases.

Studies in rat primary microglia and BV2 mouse microglia cell lines have shown that scutellarin inhibits LPSinduced nuclear translocation and DNA binding activity of $\mathrm{NF}-\kappa \mathrm{B}$, accompanied by reduced production of proinflammatory mediators, such as TNF- $\alpha$ and IL-1 $\beta$ [11]. Furthermore, recent reports have demonstrated the protective effects of scutellarin in the brain and heart of ischemic rats $[16,33]$. In line with these results, the present study found that scutellarin decreased hypertension-mediated neuronal apoptosis, possibly resulting from reduced TLR4- and NF$\kappa \mathrm{B}$-mediated production of the proinflammatory cytokines.

Scutellarin is a small molecule, and its neuroprotective effects have been well documented in different brain disease models $[13,33]$. The present study further demonstrated its anti-inflammatory and antiapoptotic action in the hypertensive brain. However, the precise molecular mechanism by which scutellarin protects against hypertension-induced brain damage still remains elusive. Further study on the protective molecular mechanisms, pharmacokinetics and brain penetration of scutellarin will help explain its limited effects on blood pressure and provide relevant evidence for future clinical applications.

\section{Conclusions}

In summary, chronic hypertension significantly enhanced the expression of TLR $4, \mathrm{NF}-\kappa \mathrm{B}$, and the production of the proinflammatory cytokines, TNF- $\alpha$, IL- $1 \beta$, and IL-18 in brains of hypertensive rats. Scutellarin lowered blood pressure and provided neuroprotective effects by suppressing TLR4/NF$\kappa \mathrm{B}$-mediated inflammation. Therefore, scutellarin may have therapeutic potential against hypertension-associated diseases.

\section{Conflict of Interests}

The authors have no conflict of interests to disclose.

\section{Authors' Contributions}

Xingyong Chen and Xiaogeng Shi contributed to in vivo experiments, RHR model, Scutellarin administration, immunofluorescent labeling, tissue preparation, western blot analyses, statistical analyses, and first draft of paper. Simei Long contributed to western blot analyses. Xiaogeng Shi and Zhong Pei contributed to design of study, conception of study, revision of paper, and final approval of paper. Ruxun Huang: design of study, financial support, revision of paper, final approval of paper. Xu Zhang, Huixin Lei, and Huanxing $\mathrm{Su}$ contributed to revision of paper. All authors read and approved the final paper. Xingyong Chen and Xiaogeng Shi contributed equally to this work.

\section{Acknowledgments}

This study was supported by the grants from the National Basic Research Program of China (30471917), the Doctoral Program of Higher Education of China (20110171110058), the Guangdong Technological grant (2011B050400031), and the Guangdong Province Natural Science Fund (Doctoral Initiating Project, 9451040701003684).

\section{References}

[1] J. Y. Park, J. W. Yun, Y. W. Choi et al., "Antihypertensive effect of gomisin A from Schisandra chinensis on angiotensin II-induced hypertension via preservation of nitric oxide bioavailability," Hypertension Research, vol. 35, no. 9, pp. 928-934, 2012.

[2] C. Díaz-Ruiz, J. Wang, H. Ksiezak-Reding et al., "Role of hypertension in aggravating abeta neuropathology of ad type and taumediated motor impairment," Cardiovascular Psychiatry and Neurology, vol. 2009, Article ID 107286, 9 pages, 2009.

[3] D. Carnevale, G. Mascio, M. A. Ajmone-Cat et al., "Role of neuroinflammation in hypertension-induced brain amyloid pathology," Neurobiology of Aging, vol. 33, no. 1, pp. 205-219, 2012.

[4] Y. Kong and Y. Le, "Toll-like receptors in inflammation of the central nervous system," International Immunopharmacology, vol. 11, no. 10, pp. 1407-1414, 2011.

[5] A. Shastri, D. M. Bonifati, and U. Kishore, "Innate immunity and neuroinflammation," Mediators Inflamm, vol. 2013, no. 6, Article ID 342931, 19 pages, 2013.

[6] J.-J. Jin, H.-D. Kim, J. A. Maxwell, L. Li, and K.-I. Fukuchi, "Toll-like receptor 4-dependent upregulation of cytokines in a transgenic mouse model of Alzheimer's disease," Journal of Neuroinflammation, vol. 5, article 23, 2008.

[7] S. Lin, Q. Yin, Q. Zhong et al., "Heme activates TLR4-mediated inflammatory injury via MyD88/TRIF signaling pathway in intracerebral hemorrhage," Journal of Neuroinflammation, vol. 9, article 46, 2012.

[8] R. Eiler, C. Schmaderer, K. Rusai et al., "Hypertension augments cardiac Toll-like receptor 4 expression and activity," Hypertension Research, vol. 34, no. 5, pp. 551-558, 2011.

[9] Y. Wang, P. Ge, and Y. Zhu, "TLR2 and TLR4 in the brain injury caused by cerebral ischemia and reperfusion," Mediators of Inflammation, vol. 2013, Article ID 124614, 8 pages, 2013.

[10] M. Song, J. Jin, J.-E. Lim et al., “TLR4 mutation reduces microglial activation, increases $\mathrm{A} \beta$ deposits and exacerbates cognitive deficits in a mouse model of Alzheimer's disease," Journal of Neuroinflammation, vol. 8, article 92, 2011.

[11] S. Wang, H. Wang, H. Guo, L. Kang, X. Gao, and L. Hu, "Neuroprotection of Scutellarin is mediated by inhibition of microglial inflammatory activation," Neuroscience, vol. 185, pp. 150-160, 2011.

[12] H. Liu, X. Yang, R. Tang, J. Liu, and H. Xu, "Effect of scutellarin on nitric oxide production in early stages of neuron damage induced by hydrogen peroxide," Pharmacological Research, vol. 51, no. 3, pp. 205-210, 2005. 
[13] L. Qian, M. Shen, H. Tang et al., "Synthesis and protective effect of scutellarein on focal cerebral ischemia/reperfusion in rats," Molecules, vol. 17, no. 9, pp. 10667-10674, 2012.

[14] H. Hong and G.-Q. Liu, "Scutellarin protects PC12 cells from oxidative stress-induced apoptosis," Journal of Asian Natural Products Research, vol. 8, no. 6, pp. 471-479, 2006.

[15] H. Guo, L.-M. Hu, S.-X. Wang et al., "Neuroprotective effects of scutellarin against hypoxic-ischemic-induced cerebral injury via augmentation of antioxidant defense capacity," Chinese Journal of Physiology, vol. 54, no. 6, pp. 399-405, 2011.

[16] H.-F. Zhang, X.-M. Hu, L.-X. Wang, S.-Q. Xu, and F.-D. Zeng, "Protective effects of scutellarin against cerebral ischemia in rats: evidence for inhibition of the apoptosis-inducing factor pathway," Planta Medica, vol. 75, no. 2, pp. 121-126, 2009.

[17] H. Dai, J. Gu, L.-Z. Li, L.-M. Yang, H. Liu, and J.-Y. Li, “Scutellarin benzyl ester partially secured the ischemic injury by its anti-apoptosis mechanism in cardiomyocytes of neonatal rats," Journal of Chinese Integrative Medicine, vol. 9, no. 9, pp. 10141021, 2011.

[18] J.-Y. Li, H. Dai, H. Liu, J. Gu, and L.-Z. Li, "Effects of scutellarin benzyl ester on the expressions of Bcl-2 and Bax in cardiomyocytes injured by acute hypoxia," Chinese Critical Care Medicine, vol. 23, no. 6, pp. 337-340, 2011.

[19] Z.-H. Tan, L.-H. Yu, H.-L. Wei, and G.-T. Liu, "Scutellarin protects against lipopolysaccharide-induced acute lung injury via inhibition of NF- $\kappa \mathrm{B}$ activation in mice," Journal of Asian Natural Products Research, vol. 12, no. 3, pp. 175-184, 2010.

[20] S. Wang, H. Wang, H. Guo, L. Kang, X. Gao, and L. Hu, "Neuroprotection of Scutellarin is mediated by inhibition of microglial inflammatory activation," Neuroscience, vol. 185, pp. 150-160, 2011.

[21] Z. Xiong, C. Liu, F. Wang et al., "Protective effects of breviscapine on ischemic vascular dementia in rats," Biological and Pharmaceutical Bulletin, vol. 29, no. 9, pp. 1880-1885, 2006.

[22] J. Zeng, Y. Zhang, J. Mo, Z. Su, and R. Huang, "Two-kidney, two clip renovascular hypertensive rats can be used as stroke-prone rats," Stroke, vol. 29, no. 8, pp. 1708-1714, 1998.

[23] C. Cui, A. F. Chen, Z. Jiang et al., "Inhibition of $\mathrm{NAD}(\mathrm{P}) \mathrm{H}$ oxidase reduces fibronectin expression in stroke-prone renovascular hypertensive rat brain," Clinical and Experimental Pharmacology and Physiology, vol. 34, no. 4, pp. 304-309, 2007.

[24] H. Sui, W. Wang, P. H. Wang, and L. S. Liu, "Protective effect of antioxidant ebselen (PZ51) on the cerebral cortex of strokeprone spontaneously hypertensive rats," Hypertension Research, vol. 28, no. 3, pp. 249-254, 2005.

[25] E. Yamamoto, N. Tamamaki, T. Nakamura et al., "Excess salt causes cerebral neuronal apoptosis and inflammation in stroke-prone hypertensive rats through angiotensin II-induced NADPH oxidase activation," Stroke, vol. 39, no. 11, pp. 30493056, 2008.

[26] E. Yamamoto, T. Yamashita, T. Tanaka et al., "Pravastatin enhances beneficial effects of olmesartan on vascular injury of saltsensitive hypertensive rats, via pleiotropic effects," Arteriosclerosis, Thrombosis, and Vascular Biology, vol. 27, no. 3, pp. 556-563, 2007.

[27] T. Wu, H. Wu, J. Wang, and J. Wang, "Expression and cellular localization of cyclooxygenases and prostaglandin E synthases in the hemorrhagic brain," Journal of Neuroinflammation, vol. 8, article 22, 2011.
[28] S. Xing, Y. Zhang, J. Li et al., "Beclin 1 knockdown inhibits autophagic activation and prevents secondary neurodegenerative damage in the ipsilateral thalamus following focal cerebral infarction," Autophagy, vol. 8, no. 1, pp. 63-76, 2012.

[29] G. F. Bomfim, R. A. Dos Santos, M. A. Oliveira et al., “Tolllike receptor 4 contributes to blood pressure regulation and vascular contraction in spontaneously hypertensive rats," Clinical Science, vol. 122, no. 11, pp. 535-543, 2012.

[30] S. Walter, M. Letiembre, Y. Liu et al., "Role of the toll-like receptor 4 in neuroinflammation in Alzheimer's disease," Cellular Physiology and Biochemistry, vol. 20, no. 6, pp. 947-956, 2007.

[31] J. R. Caso, J. M. Pradillo, O. Hurtado, P. Lorenzo, M. A. Moro, and I. Lizasoain, "Toll-like receptor 4 is involved in brain damage and inflammation after experimental stroke," Circulation, vol. 115, no. 12, pp. 1599-1608, 2007.

[32] F. Hua, J. Ma, T. Ha et al., "Activation of Toll-like receptor 4 signaling contributes to hippocampal neuronal death following global cerebral ischemia/reperfusion," Journal of Neuroimmunology, vol. 190, no. 1-2, pp. 101-111, 2007.

[33] L.-L. Lin, A.-J. Liu, J.-G. Liu, X.-H. Yu, L.-P. Qin, and D.-F. Su, "Protective effects of scutellarin and breviscapine on brain and heart ischemia in rats," Journal of Cardiovascular Pharmacology, vol. 50, no. 3, pp. 327-332, 2007. 


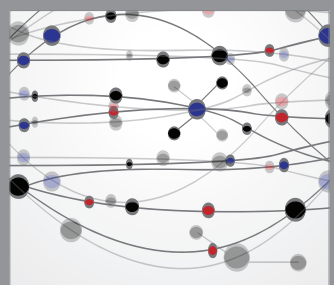

The Scientific World Journal
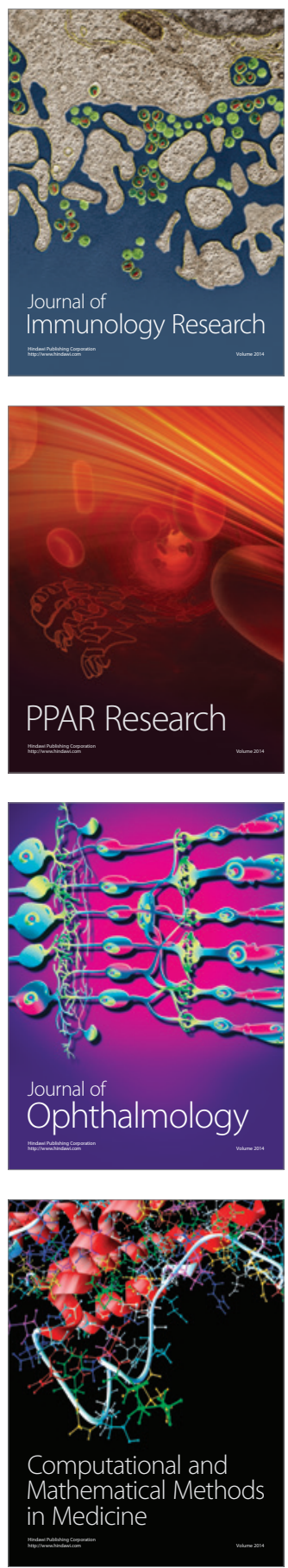

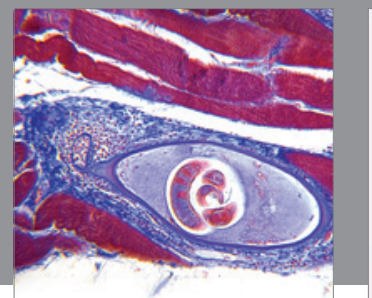

Gastroenterology

Research and Practice
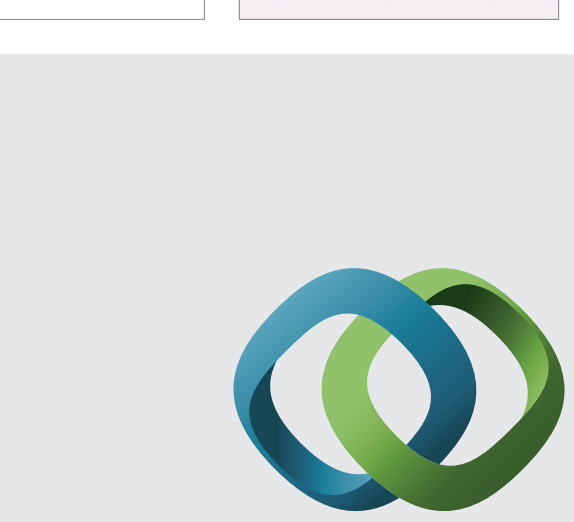

\section{Hindawi}

Submit your manuscripts at

http://www.hindawi.com
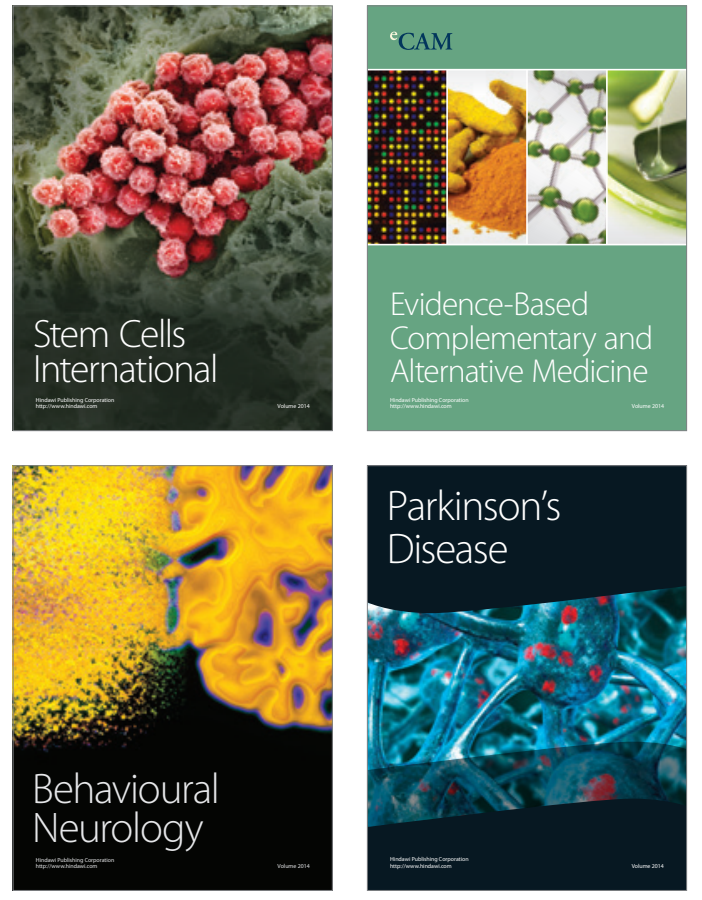
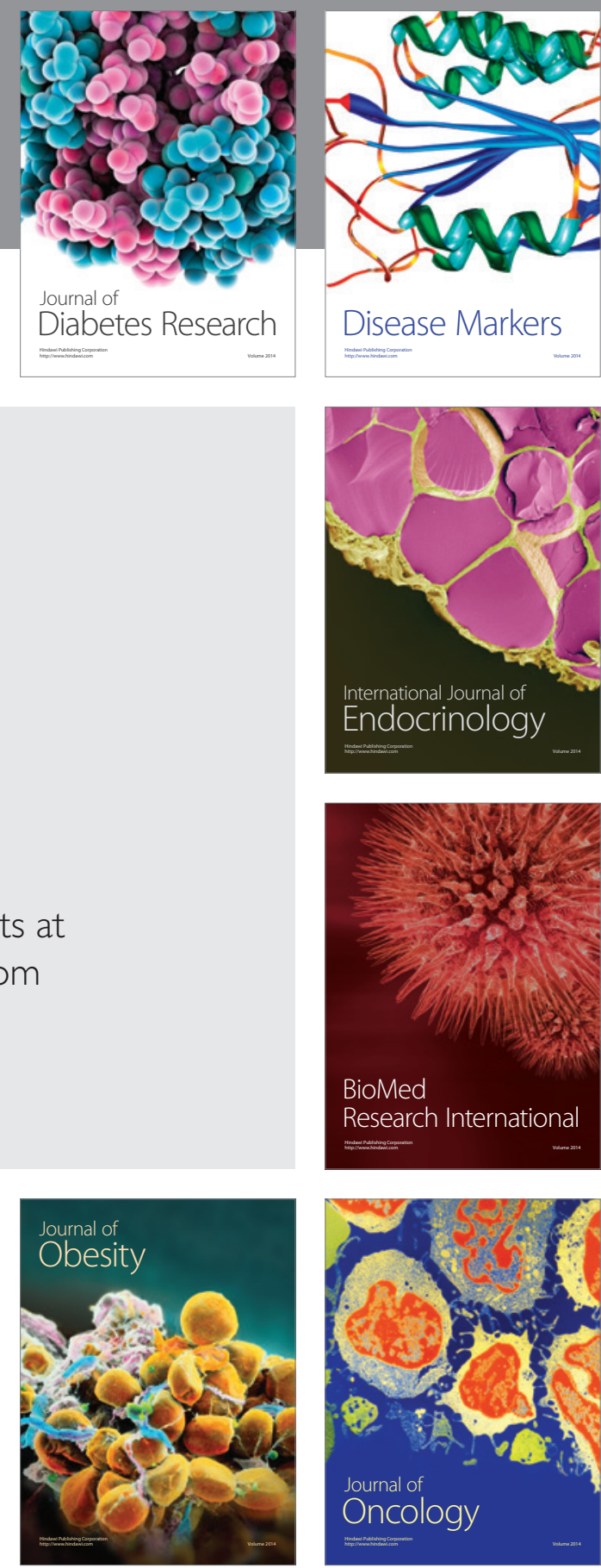

Disease Markers
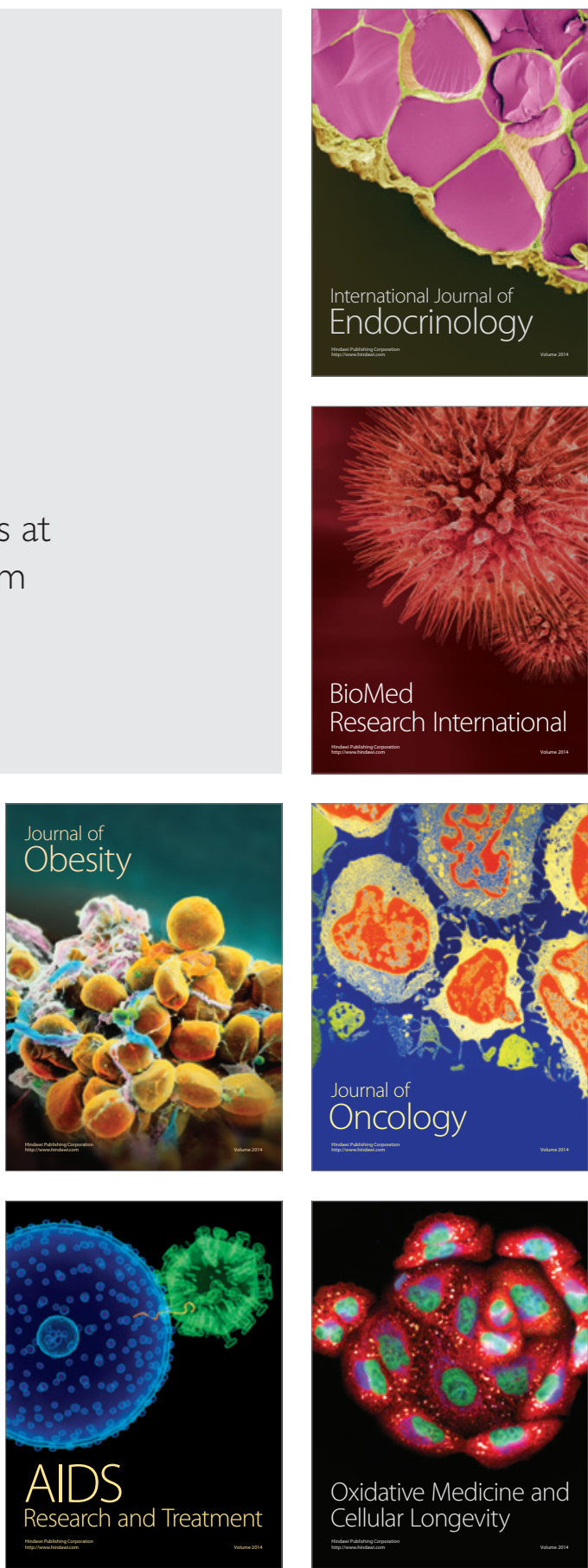